\title{
A THEOREM ON LOCAL ISOMETRIES
}

\author{
W. A. KIRK
}

A mapping $\phi$ of a $G$-space $R$ (Busemann [1]) on itself is a locally isometric mapping if for each $x \in R$ there is a number $\eta_{x}>0$ such that $\phi$ maps the spherical neighborhood $S\left(x, \eta_{x}\right)$ isometrically on $S\left(\phi(x), \eta_{x}\right)$. The problem we are concerned with is that of determining conditions on a $G$-space $R$ under which every locally isometric mapping of $R$ on itself is an isometry. Several such conditions have recently been given by Busemann $[1, \S 27],[2]$, Szenthe [4], [5], and the author [3]. In this paper we are concerned with the more general of the conditions given by Szenthe [5].

For a fixed point $p \in R$, consider the collection $G(p)$ of all geodesic curves which begin and end at $p$, and which do not contain subarcs traversed more than once. For $h \in G(p)$, let $l(h)$ denote the length of $h$. Let $\lambda_{i}(p)$ and $\lambda_{s}(p)$ equal, respectively, inf $l(h)$ and $\sup l(h)$ for all $h \in G(p)$. Put $\lambda_{i}(p)=\infty$ and $\lambda_{s}(p)=0$ if $G(p)$ is empty. Let

$$
\lambda_{i}=\inf _{p \in R} \lambda_{i}(p) ; \quad \lambda_{s}=\sup _{p \in R} \lambda_{s}(p) .
$$

Szenthe has proved [5] that if $\lambda_{i}>0$ and $\lambda_{s}<\infty$, then every locally isometric mapping of $R$ on itself is an isometry.

Szenthe's condition provides a solution to a problem suggested by Busemann [1, p. 405] in that it applies to a cylinder with euclidean metric. It is known [1] that every locally isometric mapping of a compact $G$-space on itself is an isometry. Szenthe's condition, however, fails to hold in every compact space since, as he points out [5, p. 441], $\lambda_{s}=\infty$ on a torus with euclidean metric. Our purpose here is to present a condition more general than Szenthe's which holds in every compact space.

Let $\phi$ denote a locally isometric mapping of a $G$-space $R$ on itself. Let $\rho(p)$ be the supremum of those numbers $\rho$ such that if $x, y$ are in the spherical neighborhood $S(p, \rho), x \neq y$, then there exists a point $z \neq y$ such that $x y+y z=x z$. We shall make use of the following properties of $\phi$ which are found in Busemann $[1, \S 27]$.

(1) If $\phi$ is 1-1 then $\phi$ is an isometry.

(2) If $\phi\left(p_{1}\right)=\phi\left(p_{2}\right)=p, p_{1} \neq p_{2}$, then $p_{1} p_{2} \geqq 2 \rho(p)$.

(3) The number of points of $R$ which lie over a given point of $R$ is countable, and is the same for different points of $R$.

Presented to the Society, January 25, 1966; received by the editors April 13, 1965. 
The following fact is an easy consequence of propositions (27.4) and (27.11) of Busemann [1].

(4) If $a, b \in R$ are given, and if $\phi(\bar{a})=a$, then there is a point $\bar{b} \in R$ such that $\phi(\bar{b})=b$ and $\bar{a} \bar{b}=a b$.

Definition. A geodesic curve $h \in G(p)$ is called a circular loop at $p$ provided there exists a $z$ such that

$$
h=T_{1}(p, z) \cup T_{2}(p, z)
$$

where $T_{1}(p, z)$ and $T_{2}(p, z)$ denote (necessarily) distinct metric segments with endpoints $p$ and $z$.

Let $Q(p)$ denote the collection of all circular loops at $p$. For $h \in Q(p)$, let $l(h)$ denote the length of $h$. Further, let

$$
\begin{aligned}
& l_{i}(p)=\inf [l(h): h \in Q(p)], \\
& l_{s}(p)=\sup [l(h): h \in Q(p)],
\end{aligned}
$$

If $Q(p)=\varnothing$, take $l_{i}(p)=\infty$ and $l_{s}(p)=0$. Then let

$$
\begin{aligned}
& l_{i}=\inf \left[l_{i}(p): p \in R\right], \\
& l_{s}=\sup \left[l_{s}(p): p \in R\right] .
\end{aligned}
$$

It will be helpful to establish the following lemma before turning to the theorem.

Lemma. Let $\phi$ be a locally isometric mapping of $R$ on itself, and suppose that $\phi\left(p_{1}\right)=p$. Let

$$
W=\left\{x \in R: \phi(x)=p, x \neq p_{1}\right\} .
$$

If $W \neq \varnothing$ then there is at least one point $p_{2} \in W$ such that $p_{1} p_{2}$ $=\inf \left[p_{1} x: x \in W\right]$. Further, if $T\left(p_{1}, p_{2}\right)$ is a segment joining $p_{1}$ and $p_{2}$, then $\phi\left(T\left(p_{1}, p_{2}\right)\right) \in Q(p)$.

Proof. The first part of the conclusion follows easily. If $x, y \in W$, $x \neq y$, then $x y \geqq 2 \rho(p)$ by (2). Hence by finite compactness of $R$, the set of points $x \in W$ such that $p_{1} x$ is less than a given number is finite.

We now prove that $\phi\left(T\left(p_{1}, p_{2}\right)\right) \in Q(p)$. Let $z_{1}$ denote the midpoint of $T\left(p_{1}, p_{2}\right)$. By (4), there is a point $p_{3}$ such that $\phi\left(p_{3}\right)=p$ and $z_{1} p_{3}=z p$, where $z=\phi\left(z_{1}\right)$. If $p_{3}=p_{1}$ then $p z=p_{1} z_{1}=(1 / 2) p_{1} p_{2}$. Otherwise

$$
p_{1} p_{2}=p_{1} z_{1}+z_{1} p_{2} \geqq p_{1} z_{1}+z p=p_{1} z_{1}+z_{1} p_{3} \geqq p_{1} p_{3} \geqq p_{1} p_{2},
$$

and thus in either case $p z=p_{2} z_{1}=(1 / 2) p_{1} p_{2}$.

Let $T\left(p_{1}, z_{1}\right)$ and $T\left(z_{1}, p_{2}\right)$ be subsegments of $T\left(p_{1}, p_{2}\right)$. Then $\phi\left(T\left(p_{1}, z_{1}\right)\right)$ and $\phi\left(T\left(z_{1}, p_{2}\right)\right)$ are segments since each is a geodesic arc whose length is equal to the distance between its endpoints. Therefore, 


$$
\phi\left(T\left(p_{1}, p_{2}\right)\right)=\phi\left(T\left(p_{1}, z_{1}\right)\right) \cup \phi\left(T\left(z_{1}, p_{2}\right)\right) \in Q(p) .
$$

THEOREM. If $R$ is a $G$-space for which $l_{i}>0$ and $l_{s}<\infty$, then every locally isometric mapping of $R$ on itself is an isometry.

Proof. Let $\phi$ be a locally isometric mapping of $R$ on itself and suppose that $\phi$ is not an isometry. Let $p \in R$. We define the sequence $\left\{p_{n}\right\}$ as follows. By (1) $\phi$ is not $1-1$, so by the lemma, for each positive integer $n$ there is a point $p_{n}$ such that $\phi\left(p_{n}\right)=\phi^{n+1}(p)$ and

$$
p_{n} \phi^{n}(p)=\inf \left[x \phi^{n}(p): \phi(x)=\phi^{n+1}(p), x \neq \phi^{n}(p)\right] .
$$

Using (4) and the fact that $\phi^{n}$ is also a local isometry, let $p_{n}$ be a point such that $\phi^{n}\left(p_{n}\right)=p_{n}$ and $p p_{n}=\phi^{n}(p) p_{n}$.

By the lemma $\phi$ maps $T\left(\phi^{n}(p), p_{n}\right)$ into an element of $Q\left(\phi^{n+1}(p)\right)$, which has length $\phi^{n}(p) p_{n}$, and hence $p p_{n} \leqq l_{s}$. Further, $p_{m} \neq p_{n}$ if $n \neq m$ since, assuming $n<m, \phi^{m}\left(p_{m}\right)=p_{m}$ while $\phi^{m}\left(p_{n}\right)=\phi^{m}(p) \neq p_{m}$. Therefore, since $R$ is finitely compact, there are integers $k, l$ such that $p_{k} p_{l}<l_{i}$ where $k \neq l$. Assume $k<l$. Then $\phi^{l}\left(p_{l}\right)=p_{l}$ and $\phi^{l}\left(p_{k}\right)$ $=\phi^{l}(p)$. Thus $p_{l} \phi^{l}(p) \leqq p_{l} p_{k}<l_{i}$. Since $T\left(\phi^{l}(p), p_{l}\right)$ is mapped by $\phi$ into an element of $Q\left(\phi^{l+1}(p)\right)$ which has length $p_{l} \phi^{l}(p)$, we have a contradiction.

That our theorem is more general than Szenthe's is evident; the collection $Q(p)$ is contained in the collection $G(p)$. It is easily seen that our condition also holds in all compact spaces. An element of $Q(p)$ is composed of two distinct segments $T_{1}(p, z)$ and $T_{2}(p, z)$.In a compact space the lengths of such segments are bounded above by the diameter of the space and bounded below by inf $[\rho(p): p \in R]$ which is positive (cf. [1, p. 39]).

\section{REFERENCES}

1. H. Busemann, The geometry of geodesics, Academic Press, New York, 1955.

2. - Geometries in which the planes minimize area, Ann. Mat. Pura Appl. 55 (1961), 171-189.

3. W. A. Kirk, On locally isometric mappings of a G-space on itself, Proc. Amer. Math. Soc. 15 (1964), 584-586.

4. J. Szenthe, Über lokalisometrische Abbildungen von G-Räumen auf sich, Ann. Mat. Pura Appl. 55 (1961), 37-46.

5. - Über metrische Räume, deren lokalisometrische Abbildungen Isometrien sind, Acta Math. Acad. Sci. Hungar. 13 (1962), 433-441.

University of California, Riverside 